\author{
Nota científica \\ (Short communication)
}

\title{
PRIMEROS REGISTROS DEL CHIPE DORADO PROTONOTARIA CITREA (AVES: PASSERIFORMES) EN EL ESTADO DE PUEBLA, MÉXICO
}

\author{
Francisco JaVier JiMÉNEZ MORENO, ARTURo DUCHATEAU, JoSÉ ANTONIO GONZÁLEZ OREJA* \\ Facultad de Ciencias Biológicas. BUAP, Benemérita Universidad Autónoma de Puebla. Edificio 112-A, Ciudad Universitaria, \\ 72570 Puebla, México. <pacosaurus1@gmail.com>; <arturoduchateau@gmail.com>; >jgonzorj@ hotmail.com> \\ *Autor de correspondencia: <jgonzorj@hotmail.com>
}

Recibido: 09/05/2018; aceptado: 15/11/2018; publicado en línea: 10/04/2019

Editor responsable: Octavio Rojas Soto

\begin{abstract}
Jiménez Moreno, F. J., Duchateau, A., González Oreja, J. A. (2019) Primeros registros del Chipe dorado Protonotaria citrea (Aves: Passeriformes) en el estado de Puebla, México. Acta Zoológica Mexicana (nueva serie), 35, 1-5. https://doi.org/10.21829/azm.2019.3501204
\end{abstract}

RESUMEN. Durante la temporada de reproducción, el Chipe dorado (Protonaria citrea) se puede encontrar desde el este de Estados Unidos y Canadá, mientras que en invierno se extiende desde el sur de México hasta América Central y el Caribe. Se ha reportado a esta especie como transitoria en el Eje Neovolcánico Transversal. En esta nota informamos sobre los dos primeros registros de la especie en el estado de Puebla, México. El 18 de abril de 2017, observamos un macho en la ex Hacienda de Chautla $\left(19^{\circ} 18^{\prime} 55^{\prime \prime}\right.$ N,

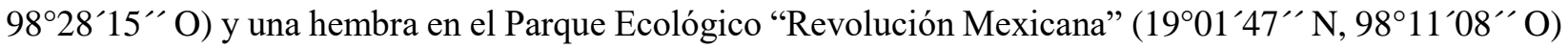
en la ciudad de Puebla. Nuestros datos, y los registros previos de la especie en la Ciudad de México, podrían sugerir que el Chipe dorado puede aprovechar las condiciones ambientales y/o recursos naturales asociados con los ecosistemas urbanos modernos. Por otro lado, estos registros también podrían ser el resultado del creciente interés por las actividades de observación de aves en entornos urbanos. Se requieren más trabajos de campo para mejorar nuestro conocimiento de la distribución del Chipe dorado en México y determinar si es un migrante transitorio en Puebla o puede considerarse un residente de invierno.

Jiménez Moreno, F. J., Duchateau, A., González Oreja, J. A. (2019) First records of the Prothonotary Warbler Protonotaria citrea (Aves: Passeriformes) in the state of Puebla, Mexico. Acta Zoológica Mexicana (nueva serie), 35, 1-5. https://doi.org/10.21829/azm.2019.3501204

ABSTRACT: During the breeding season, the Prothonotary Warbler (Protonotaria citrea) can be found through the Middle East of the United States and Canada, whereas, in winter, it ranges from southern Mexico to Central America and the Caribbean. It has been reported as a transient species in the Trans-Mexican Volcanic Belt. In this note we report on the first two records of the species in the state of Puebla, central

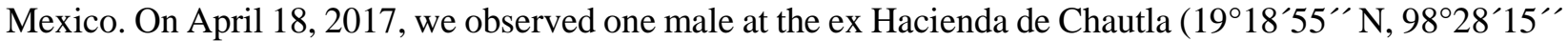

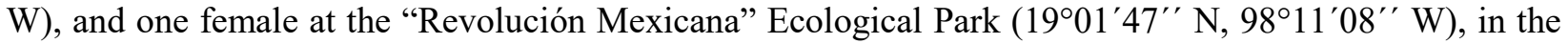
city of Puebla. Our data, and previous records of the species in Mexico City, could suggest that the Prothonotary Warbler can take benefit of the environmental conditions and/or natural resources associated with modern urban ecosystems. On the other hand, these records could also be the result of the increasing interest for bird-watching activities in urban settings. More field-work is needed to improve our knowledge 
of the distribution of the Prothonotary Warbler in Mexico during migration, and to ascertain if the species is a transient migrant in Puebla or can be considered as a winter resident.

El Chipe dorado (Protonaria citrea) es un pequeño parúlido que, en su etapa reproductiva, se distribuye en la Región Neártica (este de Estados Unidos y Canadá, donde se considera una especie amenazada). Durante la temporada de cría habita principalmente en áreas cubiertas de vegetación leñosa de baja altura, frecuentemente con cuerpos de agua (Kaufman, 2005; Hoover, 2009; Alderfer, 2014). Migra a través del Golfo de México hasta Colombia, Venezuela y otros países del Caribe, en donde pasa el invierno (Petit, 1999; Alderfer, 2014; véase también el mapa de distribución en IUCN, 2018). En México puede registrarse sobre todo en la vertiente del Golfo y la península de Yucatán, y se considera una especie transitoria en el Eje Neovolcánico Transversal (Howell \& Webb, 1995; Navarro Sigüenza et al., 2007).

Es un chipe fácil de identificar: el macho adulto presenta la cabeza, nuca, garganta, pecho y vientre, de un brillante color amarillo-dorado; el dorso es verdoso y las cobertoras inferiores caudales son blancas. Las alas son de color azul-grisáceo; y los ojos y el pico, negro intenso. Aunque el dimorfismo sexual no es acentuado, la hembra es un poco más opaca, con el píleo y la nuca de color verde-oliva (Kaufman, 2005; Alderfer, 2014).

El 18 de abril de 2017, a las 10:00 a.m., observamos un macho de Chipe dorado (Fig. 1) en las proximidades de la ex Hacienda de Chautla $\left(19^{\circ} 18^{\prime} 55^{\prime \prime} \mathrm{N}, 98^{\circ} 28^{\prime} 15^{\prime \prime} \mathrm{O}\right)$, en el Municipio de San Lucas el Grande, estado de Puebla. La ex Hacienda de Chautla se sitúa a unos 2,300 msnm, en una zona de transición entre el clima semifrío de la Sierra Nevada y el clima templado del Valle de Puebla. Está rodeada por una orla de vegetación forestal y, a mayor distancia, por un mosaico agrícola con áreas urbanas en expansión. El individuo observado se encontraba siempre a muy baja altura, alternando entre la vegetación herbácea y la base de algunos árboles, y en ocasiones muy cerca de la orilla del cuerpo de agua junto a la ex Hacienda.

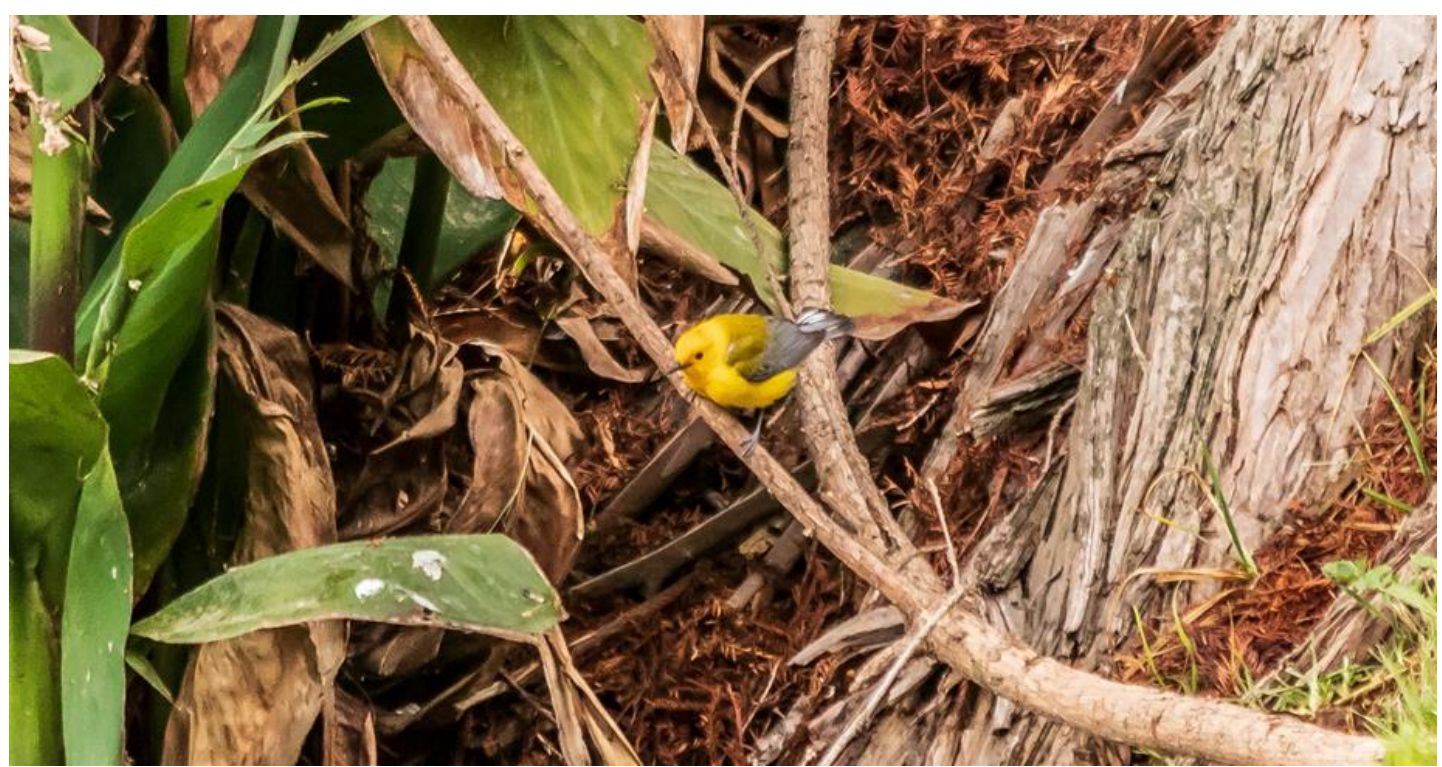

Figura 1. Macho de Chipe dorado (Protonotaria citrea), observado a nivel del suelo en la exHacienda de Chautla, San Lucas el Grande, estado de Puebla. Imagen capturada el 18 de abril de 2017, a las 10:05 a.m., con una Canon EOS 80D. (CArturo Duchateau. 
El mismo día 18 de abril, entre las 16:30 y las 16:55 horas, observamos una hembra de Chipe dorado (Fig. 2) a unos $43.5 \mathrm{~km}$ de la ex Hacienda de Chautla, en el Parque Ecológico "Revolución Mexicana" $\left(19^{\circ} 01^{\prime} 47^{\prime \prime} \mathrm{N}, 98^{\circ} 11^{\prime} 08^{\prime \prime} \mathrm{O}\right)$, dentro de la superficie urbana de la ciudad de Puebla, que se sitúa a unos 2,100 msnm y cuyo clima es templado sub-húmedo, con lluvias en verano. El individuo observado estaba forrajeando cerca de la orilla de uno de los cuerpos de agua del Parque Ecológico, entre las ramas de una bugambilia (Bougainvillea glabra) de porte bajo, a menos de $1 \mathrm{~m}$ del suelo.

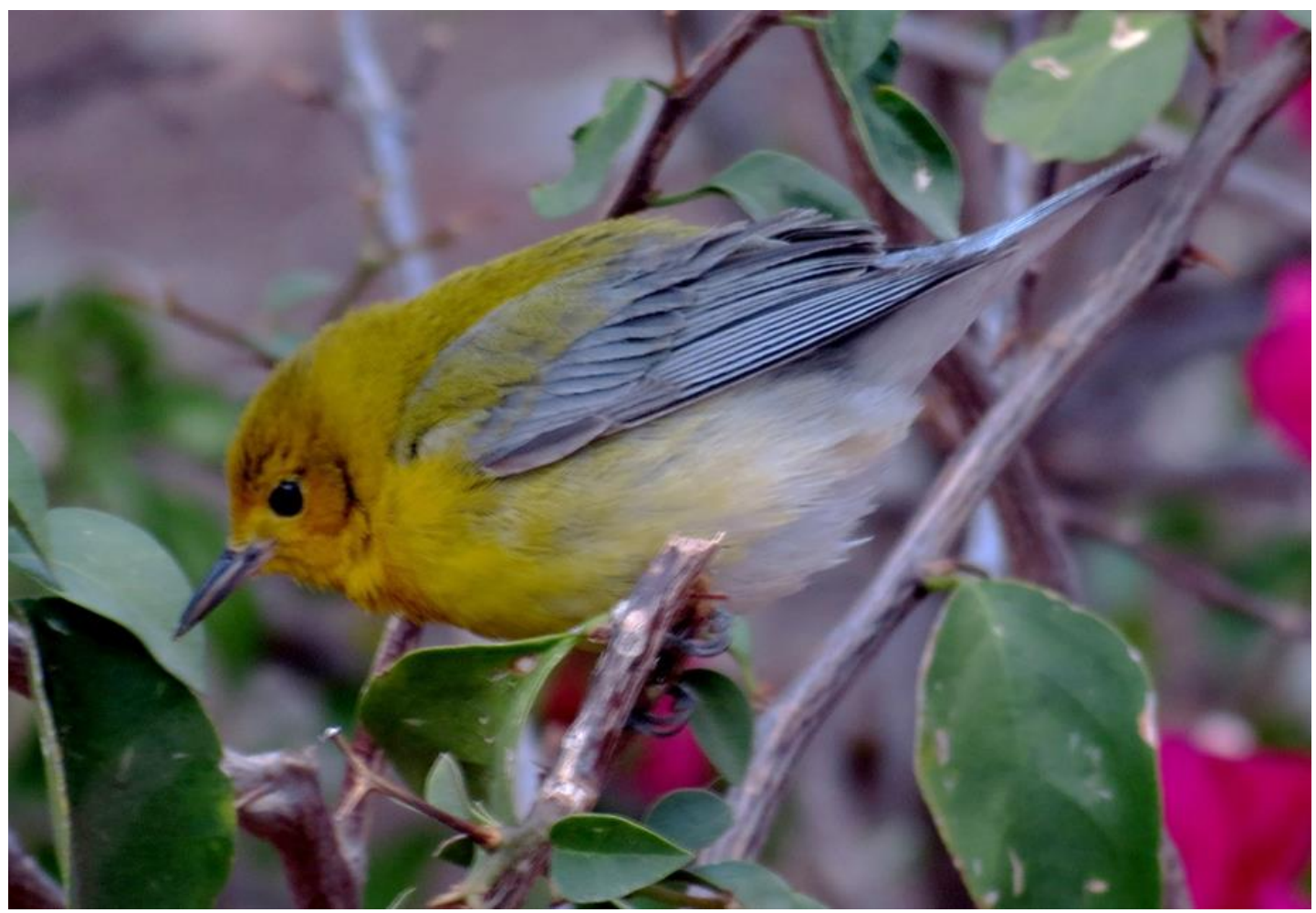

Figura 2. Hembra de Chipe dorado (Protonotaria citrea), observada entre las ramas de una buganvilia (Bougainvillea glabra) en el Parque Ecológico "Revolución Mexicana”, Puebla, estado de Puebla. Imagen capturada el 18 de abril de 2017, a las 16:45 horas, con una Canon PowerShot SX40 HS. @ Francisco Javier Jiménez Moreno.

El Chipe dorado no aparece en el inventario de las aves del Municipio de Puebla (Mendoza Cuamatzi et al., 2012). Es más, aunque Hernández Castán et al. (2013) sí incluyeron al Chipe dorado en su listado de las aves del estado de Puebla (como una especie potencialmente presente), lo cierto es que, hasta este momento, no se conocía ningún registro. Por lo tanto, de acuerdo con los criterios prácticos propuestos por Sánchez González (2013), las observaciones que reportamos en este trabajo (Fig. 3) constituyen los dos primeros registros confirmados para el estado de Puebla.

El registro de especies fuera de su área de distribución conocida resulta importante, ya que contribuye a una mejor delimitación de la misma y permite descubrir nuevas poblaciones (Sánchez González, 2013). Además, puede arrojar luz sobre la ecología de las especies migratorias, como el Chipe dorado. González Oreja (2011) revisó 16 publicaciones científicas que informaban de la composición de las comunidades de aves presentes en 11 ecosistemas urbanos de todo México, y el Chipe dorado no había sido citado nunca en tales publicaciones. Ahora bien, el hecho de que la especie no apareciera en estos estudios no implica que no hubiera sido registrada previamente en entornos urbanos. De hecho, Varona-Graniel (2001) lo había observado ya en varias áreas verdes de la Ciudad de México: Bosque de Chapultepec y Parque Aragón (octubre, 1996) y Parque Alameda (abril, 1997). Además, DeSucre Medrano et al. (2010) informaron de la colecta de una hembra (octubre, 1996) en el Jardín Botánico de la Facultad de Estudios 
Superiores Iztacala, en Tlalnepantla, Estado de México. La aplicación web eBird (2012) recoge más observaciones históricas y recientes en áreas verdes y otros puntos de la Ciudad de México: Bosque de Chapultepec (marzo, 1987), Parque México (septiembre, 2008), Parque Triangular (Parque María Enriqueta) (agosto, 2011 y junio, 2017), Parque Ecológico de Xochimilco (septiembre, 2010 y marzo, 2017), Comisión Nacional para la Conservación de la Biodiversidad (septiembre, 2012) y Canal Nacional-Prado Churubusco (febrero y marzo, 2018).

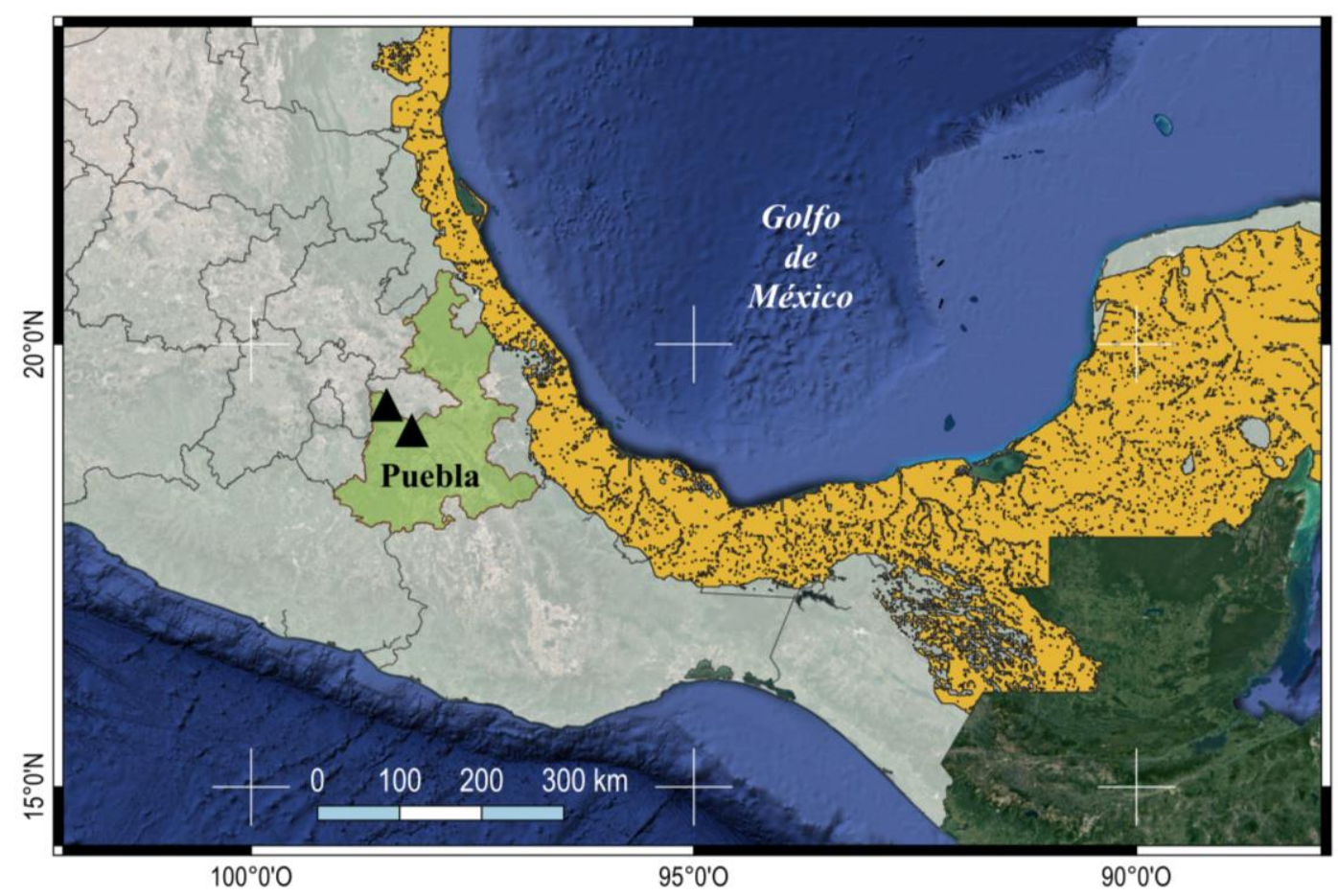

Figura 3. Distribución potencial del Chipe dorado (Protonotaria citrea) durante su migración en México (polígono irregular amarillo), según Navarro \& Peterson (2007). Se muestra la localización del estado de Puebla (polígono verde), así como la de los dos primeros registros de la especie en el estado de Puebla (este trabajo: triángulos negros).

Nuestros registros amplían el conocimiento disponible sobre la distribución del Chipe dorado en México durante la migración de primavera. A la luz de la información ya comentada, pareciera que está aumentando la frecuencia de sus registros en áreas verdes urbanas, como parques y jardines, marcadamente diferentes de los hábitats naturales en los que se reproduce en Norteamérica (Petit, 1999; Alderfer, 2014). Es posible que el Chipe dorado esté aprovechando las condiciones ambientales y/o los recursos naturales de los ecosistemas urbanos, lo que podría reflejar un proceso de urbanización. Aunque también es posible que los observadores de aves estén registrando, cada vez más, la avifauna presente en los entornos urbanos (véase Gómez de Silva \& Alvarado Reyes, 2010). En cualquier caso, es importante mejorar nuestro conocimiento sobre la distribución de esta especie en México durante la migración, así como determinar si su registro en el estado de Puebla resulta accidental o puede considerarse como residente de invierno (DeSucre Medrano et al., 2010).

AgRAdecimiEnTOS. Agradecemos el apoyo recibido de la Vicerrectoría de Investigación y Posgrado de la Benemérita Universidad Autónoma de Puebla (VIEP-BUAP; Proyecto 00625) y, sobre todo, del Programa para el Desarrollo Profesional Docente para el Tipo Superior (SEP-PRODEP; Proyecto BUAP-PTC-522). 
Tres revisores anónimos aportaron numerosas sugerencias para mejorar una versión previa de nuestro manuscrito.

\section{LITERATURA CITADA}

Alderfer, J. (2014) Complete Birds of North America. Second Edition. National Geographic, Washington, D.C., 743 pp.

DeSucre Medrano, A. E., Ramírez Bastida, P., Varona Graniel, D. E., Opengo Piña, L. H., Morlán Cahué, Y. (2010) Dos registros nuevos de aves en el Estado de México: Protonotaria citrea y Euthlypis lachrymosa. Huitzil, 11, 21-25.

eBird (2012) eBird: Una base de datos en línea para la abundancia y distribución de las aves [aplicación de internet]. eBird, Ithaca, New York. Disponible en: http://www.ebird.org. (acceso 16 de abril de 2018).

Gómez de Silva, H., Alvarado Reyes, E. (2010) Breve historia de la observación de aves en México en el siglo XX y principios del siglo XXI. Huitzil, 11 (1), 9-20.

González Oreja, J. A. (2011) Birds of different biogeographic origins respond in contrasting ways to urbanization. Biological Conservation, 144, 234-242.

Hernández Castán, J., Jiménez Moreno, F. J., Mendoza Cuamatzi, R., Rose-Burney, J., López Téllez, M. C., Torres Flores, R., Gilbert, N. (2013) Aves del Estado de Puebla. Jardín Etnobotánico Francisco Peláez R.A.C.; Peace Corps; USAID; Benemérita Universidad Autónoma de Puebla; Escuela de Biología BUAP; Instituto Nacional de Astrofísica, Óptica y Electrónica; H. Ayuntamiento de la Ciudad de Puebla y Secretaría del Medio Ambiente y Servicios Públicos, 165 pp.

Hoover, J. P. (2009) Prothonotary Warblers as indicators of hydrological conditions in bottomland forests. Pp. 128-137. In: Rich, T. D., Arizmendi, C., Demarest, D. W., Thompson, C. (Eds). Tundra to Tropics: Proceedings of the Fourth International Partners in Flight Conference. Partners in Flight, McAllen, Tex.

Howell, S. N. G., Webb, S. (1995) A Guide to the Birds of Mexico and North Central America. Oxford University Press. New York, $851 \mathrm{pp}$.

IUCN (2018) The IUCN Red List of Threatened Species: Red List Maps. http://maps.iucnredlist.org. (acceso 20 de marzo de 2018).

Kaufman, K. (2005) Field Guide to Birds of North America. Houghton Mifflin. New York, 391 pp.

Mendoza Cuamatzi, R., Rose-Burney, J., Jiménez Moreno, F. J., Escobar Pérez, V. (2012) Las Aves del Municipio de Puebla. Peace Corps, USA; CONABIO; H. Ayuntamiento Puebla y SEMARNAT. $88 \mathrm{pp}$.

Navarro, A. G., Peterson, A. T. (2007) Protonotaria citrea (Chipe dorado) Tránsito. Distribución potencial, escala 1:1000000. Museo de Zoología, Facultad de Ciencias, Universidad Nacional Autónoma de México \& University of Kansas, Museum of Natural History. México.

Navarro Sigüenza, A. G., Lira Noriega, A., Peterson, A. T., Oliveras de Ita, A., Gordillo-Martínez, A. (2007) Diversidad, endemismo y conservación de las aves. Pp. 461-483. In: Luna, I., Morrone, J. J., Espinosa, D. (Eds.). Biodiversidad de la Faja Volcánica Transmexicana. UNAM. México.

Sánchez-González, L. A. (2013) Cuando un "nuevo registro" es realmente un nuevo registro: consideraciones para su publicación. Huitzil, 14,17-21.

Petit, L. J. (1999) Prothonotary Warbler (Protonotaria citrea). The Birds of North America, Vol. 408. The Academy of Natural Sciences, Philadelphia, and The American Ornithologist's Union, Washington, D.C.

Varona Graniel, D. E. (2001) Avifauna de Áreas Verdes Urbanas del Norte de la Ciudad de México. Tesis de Maestría en Ciencias, Universidad Nacional Autónoma de México, México DF. 